\title{
Nonlinear Dynamic Analysis of a Timoshenko Beam Resting on a Viscoelastic Foundation and Traveled by a Moving Mass
}

\author{
Ahmad Mamandi ${ }^{1}$ and Mohammad H. Kargarnovin ${ }^{2}$ \\ ${ }^{1}$ Department of Mechanical Engineering, Parand Branch, Islamic Azad University, Tehran, Iran \\ ${ }^{2}$ Department of Mechanical Engineering, Sharif University of Technology, Tehran, Iran \\ Correspondence should be addressed to Ahmad Mamandi; am_2001h@yahoo.com
}

Received 15 March 2013; Accepted 2 September 2013; Published 27 February 2014

Academic Editor: Ahmet S. Yigit

Copyright (c) 2014 A. Mamandi and M. H. Kargarnovin. This is an open access article distributed under the Creative Commons Attribution License, which permits unrestricted use, distribution, and reproduction in any medium, provided the original work is properly cited.

\begin{abstract}
The dynamic response of a Timoshenko beam with immovable ends resting on a nonlinear viscoelastic foundation and subjected to motion of a traveling mass moving with a constant velocity is studied. Primarily, the beam's nonlinear governing coupled PDEs of motion for the lateral and longitudinal displacements as well as the beam's cross-sectional rotation are derived using Hamilton's principle. On deriving these nonlinear coupled PDEs the stretching effect of the beam's neutral axis due to the beam's fixed end conditions in conjunction with the von-Karman strain-displacement relations is considered. To obtain the dynamic responses of the beam under the act of a moving mass, derived nonlinear coupled PDEs of motion are solved by applying Galerkin's method. Then the beam's dynamic responses are obtained using mode summation technique. Furthermore, after verification of our results with other sources in the literature a parametric study on the dynamic response of the beam is conducted by changing the velocity of the moving mass, damping coefficient, and stiffnesses of the foundation including linear and cubic nonlinear parts, respectively. It is observed that the inclusion of geometrical and foundation stiffness nonlinearities into the system in presence of the foundation damping will produce significant effect in the beam's dynamic response.
\end{abstract}

\section{Introduction}

The topic of vibration study of structural elements such as strings, beams, plates, and shells under the act of a moving mass is of great interest and importance in the field of structural dynamics. It should be noted that the review of numerous reported studies related to the dynamic behavior of mechanical/structural systems discloses that almost linear behavior of such systems is considered. Indeed, in reality such systems inherently and naturally have nonlinear behavior, for example, due to the geometrical nonlinearity or when they are subjected to external loadings comparatively large enough. As we will see later on in the modeling of the problem, the stretching of the beam's neutral axis due to fixed ends condition adds another nonlinearity to the dynamical behavior of the system. In addition, there are some other external distinct mechanical elements having nonlinear behavior attached to such structures like shock energy absorbers or viscoelastic foundations which will add further other nonlinearities in the model analysis. From mechanical point of view, any beam structure can be modeled as a thin or thick beam for which different theories usually can be implemented. In extending the issue of the linear analysis of a Timoshenko beam resting on a viscoelastic foundation and under the act of a moving mass, certainly including the nonlinear behavior in the analysis will well provide more reliable and accurate results specifically in cases where the energy damping or any restraint on the beam's amplitude vibrations plays an important role in this filed.

Some examples of actual cases can be addressed as a bridge when traveled by moving vehicles, moving trains on railway tracks, simulation of high axial speed machining processes in milling operation, and internal fluid flow in piping systems resting on a soil foundation. Moreover, shafts in the rotating machinery resting on the elastic supports (journal bearings) and floating in an industrial lubricant can be modeled as beams on viscoelastic foundation. This approach can be viewed in modeling of rotating blades in a helicopter or in rotating blades of turbomachineries. 
In [1], the linear finite element analysis of an EulerBernoulli beam resting on an elastic foundation modeled by spring of variable stiffness subjected to moving point loads is studied. The dynamic response of an infinite Timoshenko beam on a viscoelastic foundation and under the act of a harmonic moving load has been investigated in [2]. In [3], the linear vibration and dynamic buckling of an axially loaded infinite shear beam-column on an elastic foundation and subjected to traveling loads with constant amplitude or harmonic amplitude variation with a constant velocity is studied. In this paper, formulation in the transformed field domains of time and moving space is developed and dynamic responses of the beam are obtained using Fourier transform method. The dynamic response of a simply supported Timoshenko beam resting on an elastic foundation and traveled by a moving concentrated load is obtained using method of images in [4]. In [5], the wave solution is obtained by the Laplace-transform method for a semi-infinite Timoshenko beam traveled by a concentrated moving load with a velocity which may be supersonic, intersonic, or subsonic with respect to the bending and shear-wave velocities of the beam. In [6], using Galerkin's method the dynamic stability of the transverse vibration of a simply supported Euler-Bernoulli beam resting on a viscoelastic foundation and subjected to a continuous series of equally spaced concentrated moving loads is analyzed. In this paper, the Floquet theory is utilized to study the parametric regions of stability of the beam's response. In [7], the deterministic and random dynamic responses of a nonlinear EulerBernoulli beam on an elastic foundation and under the act of a moving load are analyzed using Galerkin's method in conjunction with the dynamic finite element method. In this study, the effects of longitudinal and transverse deflections are considered in the beam's vibration analysis. In [8], a new finite element method is extracted to analyze the small amplitude vibration of a Timoshenko beam on variable two-parameter Winkler type of foundation. The solution is based on using cubic polynomial expressions for the total deflection and the bending slope of the beam. The linear dynamic analysis of transverse vibration of a simply supported Timoshenko beam resting on a Winkler type of elastic foundation and traveled by a moving concentrated mass/force is investigated in [9] by applying Galerkin's method using the mode summation technique. Besides, in [10] the linear finite element analysis of a Timoshenko beam under the act of a moving mass is conducted. The linear dynamic responses of an axially loaded damped Timoshenko beam resting on a viscoelastic foundation are studied in [11]. Recently in [12] a new analytical solution to alleviate difficulties related to Fourier analysis and numerical integration for vibration analysis of an infinite Timoshenko beam on a nonlinear viscoelastic foundation and subjected to a moving load has been studied. A new semianalytical solution for the Timoshenko beam subjected to the finite series of distributed moving loads harmonically varying in time is considered as a representation of a moving train in [13]. In [14], the dynamic response of a Rayleigh beam resting on a nonlinear viscoelastic foundation and traveled by a moving load is developed by employing the Adomian decomposition method in conjunction with coiflet expansion. An approximate closed form solution has been derived and condition for the convergence of the decomposition series has been introduced to study the dynamic response of the beam. Then, a parametric study on vibration analysis is investigated using wavelet filters of Coiflet type as a very effective tool to adapt approximating method. Transverse vibrations induced by a load moving at a constant speed along a finite or an infinite beam resting on a piece-wise homogeneous viscoelastic foundation are studied in [15]. The obtained results have direct application on the analysis of railway track vibrations induced by high-speed trains crossing regions with significantly different foundation stiffness. In [16], a numerical approach is developed to the stationary solution of an infinite Euler-Bernoulli beam on a Winkler foundation and under a moving harmonic load. In [17], a boundary element based method is applied to study the nonlinear dynamic analysis of shear deformable beams resting on tensionless nonlinear Pasternak-type viscoelastic foundation under general boundary conditions and traversed by moving loads. Analyses are performed to illustrate the accuracy of the developed method to investigate the effects of various parameters such as the load velocity, load frequency, shear deformation, foundation nonlinearity, damping, and axial compression on the beam's displacements and stresses. In [18], the issue of instability phenomenon of a three-mass oscillator uniformly moving along a continuously viscoelastic supported Timoshenko beam due to the excited Doppler waves is investigated. The proposed model corresponds to a two-level suspension vehicle running on a track and it includes the wheel/rail contact nonlinearities which contain the Hertzian contact characteristic and the possibility of contact loss. The velocities at the stability limit are calculated by means of the D-decomposition method. The characteristic equation has been obtained using Green's function of the differential operator of the Laplace transformed equations of motion. The timedomain analysis of the dynamic behavior due to the stability loss has been performed by solving the equations of motion in virtue of the convolution theorem. Moreover, the limit cycle characterizing the unstable motion is analyzed.

Based on this presumption and on the same line as the other studies this work is initiated. In the present study for the first time three nonlinear governing coupled PDEs of motion for a Timoshenko beam resting on a nonlinear viscoelastic foundation and under the act of a moving mass are derived. Then by applying Galerkin's method, these three obtained governing nonlinear coupled second order ODEs are solved numerically using Adams-Bashforth-Moulton integration method via MATLAB solver package. It should be noted that in the present study the effects of changes of different parameters on the dynamic response of the Timoshenko beam including linear and nonlinear stiffness parts of foundation, damping coefficient of viscoelastic foundation, and velocity of moving mass along with the inclusion of stretching effect of the beam's neutral axis are all considered in the dynamic analysis of the beam's deflection.

\section{Mathematical Modeling}

2.1. Problem Statement. In this study, the dynamic responses of a pinned-pinned Timoshenko beam resting on a nonlinear 


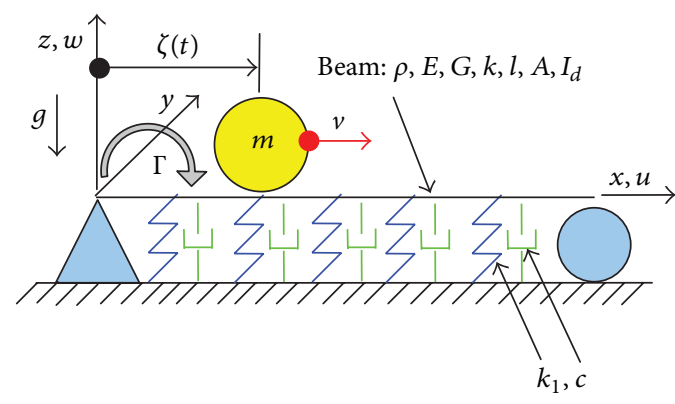

(a)

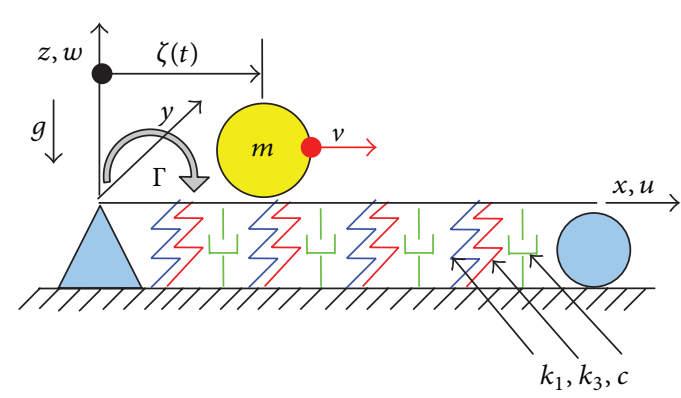

(b)

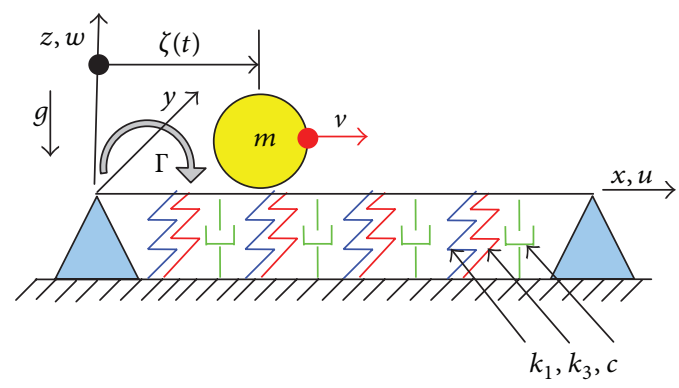

(c)

FIGURE 1: Timoshenko beam under the motion of a concentrated point mass resting on a viscoelastic foundation. (a) Timoshenko beam with no stretching effect on simply supported ends resting on a linear viscoelastic foundation, M-1 model, (b) Timoshenko beam with no stretching effect on simply supported ends resting on a nonlinear viscoelastic foundation, M-2 model, and (c) Timoshenko beam with stretching effect on pinned-pinned ends resting on a nonlinear viscoelastic foundation, M-3 model.

viscoelastic foundation and under the act of a moving mass are the main subject in our analysis. In fact our analysis focused on the study of the effects of the nonlinearities introduced due to large deflection of the beam (geometric nonlinearity) and the nonlinear stiffness of the foundation on the dynamic behaviour of above mentioned Timoshenko beam.

Consider an isotropic and homogenous Timoshenko beam with beam density $\rho$, Young's modulus $E$, shear modulus $G$, shear correction factor $k$, span length $l$, constant crosssection $A$, and diametral second cross-sectional moment $I_{d}$. To conduct our analysis, three Timoshenko beam models illustrated in Figure 1 are considered. In the first system shown in Figure 1(a), a simply supported Timoshenko beam with no stretching effect resting on a linear viscoelastic foundation with damping coefficient $c$ and linear foundation stiffness $k_{1}$ is subjected to the motion of a point mass $m$ traveling with a constant velocity $v$ along the beam length. From now on we call this model M-1. In the next system, that is, Figure 1(b), similar Timoshenko beam with the same specifications as M1 but in addition having cubic nonlinear stiffness $k_{3}$ is considered. From now on we call this model M-2. And finally the system shown in Figure 1(c) is also similar to the model M-2 but with immovable pinned-pinned end boundary conditions, that is, with stretching effect. From now on we call this model M-3. Note that in both M-2 and M-3 models, beam is resting on a nonlinear viscoelastic foundation; that is, in addition to a linear spring with stiffness of $k_{1}$ a cubic nonlinear spring with stiffness of $k_{3}$ is also included. It should be mentioned that in our upcoming analysis when the moving mass enters the left end of the beam at time $t=0$, zero initial conditions are assumed. Moreover, in our analysis it has been assumed that the moving mass during its travel never loses its contact with the beam.

2.2. Formulation. According to von-Karman's theory, the kinematics relations for the beam shown in Figure 1(b) are [19-21]

$$
\varepsilon_{x x}=u_{, x}+\frac{1}{2} w_{, x}^{2}, \quad \gamma_{x z}=w_{, x}-\Gamma, \quad \kappa_{x}=\Gamma_{, x} .
$$

Note that $u=u(x, t)$ and $w=w(x, t)$ represent the displacements of an arbitrary point located on the beam's neutral axis in the axial and the transverse, that is, the $x$ and $z$ directions, respectively, measured from the equilibrium position when unloaded. Moreover, $\Gamma=\Gamma(x, t)$ is the rotation of any arbitrary section around $y$-axis. Also, in our notation the subscripts $(, t)$ and $(, x)$ stand for the derivative with respect to the time $(t)$ and spatial coordinate $(x)$, respectively. In addition, $\varepsilon, \gamma$, and $\kappa$ are the longitudinal (or normal) strain, shear strain, and curvature at a point located on the neutral axis of the beam, respectively. To obtain the governing differential equations of motion by applying Hamilton's principle, the kinetic energy $T$ and the strain energy $U$ of the beam under consideration are [19]

$$
\begin{gathered}
T=\frac{\rho}{2} \int_{0}^{l}\left[A\left(u_{, t}{ }^{2}+w_{, t}{ }^{2}\right)+I_{d} \Gamma_{, t}{ }^{2}\right] d x, \\
U=\frac{1}{2} \int_{0}^{l}\left(E A \varepsilon_{x x}^{2}+E I_{d} \kappa_{x}{ }^{2}+k G A{\gamma_{x z}}^{2}\right) d x .
\end{gathered}
$$


Now, we can establish the Lagrangian function of the system as $L=T-\left(U-W_{e}\right)$. Applying Hamilton's principle on $L$ yields [19]

$$
\delta \int_{t_{1}}^{t_{2}} L d t=0 \Longrightarrow \delta \int_{t_{1}}^{t_{2}}(U-T) d t=\int_{t_{1}}^{t_{2}} \delta W_{e} d t .
$$

Note that the total virtual work done $\delta W_{e}$ by the nonlinear viscoelastic foundation and the traveling mass acting on the beam at the location $x=\zeta(t)$ is $[9,20,21]$

$$
\begin{aligned}
\delta W_{e}=\int_{0}^{l}[ & -\left.m u_{, t t} \delta u\right|_{x=\zeta(t)}-m\left(g+w_{, t t}+2 v w_{, x t}+v^{2} w_{, x x}\right) \\
& \left.\times\left.\delta w\right|_{x=\zeta(t)}+\left(k_{1} w+k_{3} w^{3}+c w_{, t}\right) \delta w\right] d x .
\end{aligned}
$$

After doing some mathematics, one would get the nonlinear governing coupled PDEs of motion (EOMs) as follows [9, 20, 21].

Force relation in $x$ direction:

$$
\begin{aligned}
\rho A u_{, t t} & -E A\left(u_{, x x}+w_{, x} w_{, x x}\right) \\
= & -\left.m u_{, t t}\right|_{x=\zeta(t)} \delta(x-\zeta(t)) \chi(t) .
\end{aligned}
$$

Force relation in $z$ direction:

$$
\begin{aligned}
\rho A w_{, t t}+ & k G A\left(\Gamma_{, x}-w_{, x x}\right)-E A \\
& \times\left(u_{, x x} w_{, x}+u_{, x} w_{, x x}+\frac{3}{2} w_{, x x} w_{, x}{ }^{2}\right) \\
& +k_{1} w+k_{3} w^{3}+c w_{, t} \\
= & -\left.m\left(g+w_{, t t}+2 v w_{, x t}+v^{2} w_{x x}\right)\right|_{x=\zeta(t)} \\
& \times \delta(x-\zeta(t)) \chi(t) .
\end{aligned}
$$

Moment relation about $y$ direction:

$$
\rho I_{d} \Gamma_{, t t}-E I_{d} \Gamma_{, x x}+k G A\left(\Gamma-w_{, x}\right)=0,
$$

where $E I_{d}$ and $\rho A$ are the beam's flexural rigidity and beam's mass per unit length, respectively. Furthermore, $\delta(x-\zeta(t))$ is Dirac's delta function in which $\zeta(t)$ is the instantaneous position of the moving mass traveling on the beam. In case where the mass is traveling with a constant velocity $v$, then its instantaneous position is given by $\zeta(t)=v t+x_{0}$, where $x_{0}$ represents the initial position of the mass at the start of its motion. Also, the $\chi(t)$ is the pulse function which is equal to 1 while the mass is traveling on the beam and 0 when the traveling mass is outside the beam span, that is, $\chi(t)=u(t)-$ $u(t-l / v)$, in which $u(t)$ represents the unit step function.

\section{Solution Method}

In this study Galerkin's method is chosen as a powerful mathematical tool to analyze the vibrations of a Timoshenko beam. Based on the separation of variables technique, the response of the Timoshenko beam in terms of the linear freeoscillation modes can be assumed as follows [9, 19-21]:

$$
\begin{aligned}
& u(x, t)=\sum_{j=1}^{n} \theta_{j}(x) r_{j}(t)=\boldsymbol{\Theta}^{T}(x) \mathbf{R}(t), \\
& w(x, t)=\sum_{j=1}^{n} \phi_{j}(x) p_{j}(t)=\boldsymbol{\Phi}^{T}(x) \mathbf{P}(t), \\
& \Gamma(x, t)=\sum_{j=1}^{n} \psi_{j}(x) q_{j}(t)=\Psi^{T}(x) \mathbf{Q}(t),
\end{aligned}
$$

where $\mathbf{P}(t), \mathbf{Q}(t)$, and $\mathbf{R}(t)$ are vectors of order $n$ listing the generalized coordinate $p_{j}(t), q_{j}(t)$, and $r_{j}(t)$, respectively, and $\boldsymbol{\Theta}(x), \boldsymbol{\Phi}(x)$, and $\boldsymbol{\Psi}(x)$ are some vectorial functions collecting the first $n$ mode shapes (eigen-functions) of $\theta_{j}(x), \phi_{j}(x)$, and $\psi_{j}(x)$, respectively.

In the next step primarily we substitute (8) to (10) into (5) to (7); then on the resulting relations, premultiplying both sides of (5) by $\Theta^{T}(x)$, (6) by $\Phi^{T}(x)$, and (7) by $\Psi^{T}(x)$, integrating over the interval $(0, l)$, and imposing the orthogonality along with Dirac's delta function conditions, the resulting nonlinear coupled modal equations of motion in matrix form are as follows:

$$
\begin{aligned}
\sum_{j=1}^{n}\left[\rho A \mathbf{J}_{i j}\right. & \left.+m \mathbf{O}_{i j}(t) \chi(\mathrm{t})\right] \ddot{r}_{j}(t)-E A \sum_{j=1}^{n} \mathbf{N}_{i j} r_{j}(t) \\
& -E A \sum_{j=1}^{n} \sum_{k=1}^{n} p_{\mathrm{j}}(t) \mathbf{L}_{i j k} p_{k}(t)=0, \quad i=1,2, \ldots, n,
\end{aligned}
$$

$$
\begin{aligned}
\sum_{j=1}^{n}\left[\rho A \mathbf{M}_{i j}\right. & \left.+m \mathbf{B}_{i j}(t) \chi(\mathrm{t})\right] \ddot{p}_{j}(t) \\
& +\sum_{j=1}^{n}\left[2 m v \chi(t) \mathbf{A}_{i j}(t)+c \mathbf{M}_{i j}\right] \dot{p}_{j}(t)
\end{aligned}
$$

$$
\begin{aligned}
& +k G A \sum_{j=1}^{n} \mathbf{F}_{i j} q_{j}(t) \\
& +\sum_{j=1}^{n}\left[m v^{2} \mathbf{C}_{i j}(t) \chi(\mathrm{t})-k G A \mathbf{H}_{i j}+k_{1} \mathbf{M}_{i j}\right] p_{j}(t) \\
& +k_{3} \sum_{j=1}^{n} \sum_{k=1}^{n} \sum_{l=1}^{n} p_{j}(t)\left(\mathbf{R}_{2}\right)_{i j k l} p_{k}(t) p_{l}(t) \\
& -E A\left[\sum_{j=1}^{n} \sum_{k=1}^{n} r_{j}(t) \mathbf{G}_{i j k} p_{k}(t)\right. \\
& +\sum_{j=1}^{n} \sum_{k=1}^{n} p_{j}(t)\left(\mathbf{R}_{1}\right)_{i j k} r_{k}(t) \\
& \left.+\frac{3}{2} \sum_{j=1}^{n} \sum_{k=1}^{n} p_{j}(t) \mathbf{I}_{i j k} p_{k}(t)^{2}\right]
\end{aligned}
$$




$$
\begin{gathered}
=-m g \chi(t) \mathbf{a}_{i}(t), \quad i=1,2, \ldots, n, \\
\rho I_{d} \sum_{j=1}^{n} \mathbf{S}_{i j} \ddot{q}_{j}(t)-\sum_{j=1}^{n}\left[E I_{d} \mathbf{K}_{i j}-k G A \mathbf{S}_{i j}\right] q_{j}(t) \\
\quad-k G A \sum_{j=1}^{n} \mathbf{E}_{i j} p_{j}(t)=0, \quad i=1,2, \ldots, n,
\end{gathered}
$$

in which dot mark over any parameter indicates the derivative with respect to the time $(t)$. All matrices appearing in above relations are given in the appendix. It is clear that (11) is three nonlinear coupled second-order ordinary differential equations (ODEs). The boundary conditions for a pinnedpinned Timoshenko beam are

$$
\begin{aligned}
& \text { Essential BCs: } u(0, t)=u(l, t)=0 \\
& \text { therefore: } \theta_{j}(x)=0 \quad \text { at } x=0, l \text {, } \\
& w(0, t)=w(l, t)=0 \\
& \text { therefore: } \phi_{j}(x)=0 \quad \text { at } x=0, l
\end{aligned}
$$

Natural BCs: $M_{y}(0, t)=M_{y}(l, t)=0$

$$
\text { therefore: } \psi_{j, x}(x)=0 \quad \text { at } x=0, l .
$$

Moreover, initial conditions (ICs) for the Timoshenko beam are

$$
\text { ICs: } \begin{aligned}
u(x, 0) & =u_{, t}(x, 0)=w(x, 0)=w_{, t}(x, 0) \\
& =\Gamma(x, 0)=\Gamma_{, t}(x, 0)=0 .
\end{aligned}
$$

This is typically a well-known eigen-function eigen-value problem, out of which the final result for the mode shapes of the Timoshenko beam with pinned-pinned ends is expressed as the following $[9,19-21]$ :

$$
\begin{gathered}
\theta_{j}(x)=\sqrt{2} \sin \left(\frac{j \pi x}{l}\right), \quad \phi_{j}(x)=\sqrt{2} \sin \left(\frac{j \pi x}{l}\right), \\
\psi_{j}(x)=\sqrt{2} \cos \left(\frac{j \pi x}{l}\right) \\
\text { with } j=1,2,3, \ldots, n .
\end{gathered}
$$

Now, we use (14) to calculate all matrix quantities given in the appendix. In the next step these evaluated matrices will be used in (11) and later the set of equations will be solved numerically using the Adams-Bashforth-Moulton integration method via MATLAB solver package to obtain values of $p_{j}(t)$, $q_{j}(t)$, and $r_{j}(t)$. By back substitution of $p_{j}(t), q_{j}(t)$, and $r_{j}(t)$ in (8) to (10), $u(x, t), w(x, t)$, and $\Gamma(x, t)$ can be obtained, respectively. Subsequently, after obtaining values for $u(x, t), w(x, t)$, and $\Gamma(x, t)$ the dynamic response of a pinned-pinned Timoshenko beam resting on a nonlinear viscoelastic foundation and subjected to a moving mass traveling with a constant velocity can be calculated.

\section{Verification of Results and Case Studies}

As mentioned in the introduction at the moment no specific results exist for the problem under consideration in the literature. Therefore, to verify the validity of the results obtained in this study we primarily consider some special cases by which our results can be compared with those existing in the literature.

\subsection{Verification of Results for the Linear and Nonlinear Analyses}

4.1.1. Linear Analysis. In the first attempt we set $\left(u_{, x x}+\right.$ $\left.w_{, x} w_{, x x}\right)$ and $\left(u_{, x x} w_{, x}+u_{, x} w_{, x x}+(3 / 2) w_{, x x} w_{, x}{ }^{2}\right)$ in the lefthand side of (5) and (6), respectively, equal to zero. Moreover, we take $k_{3}=0$ and $c=0$. This will lead us to a set of new relations for $u(x, t), w(x, t)$, and $\Gamma(x, t)$ representing the linear form of governing EOMs of a Timoshenko beam on a linear Winkler type of foundation. To verify the validity of the obtained results out of our analysis, we consider the data given in [9] as $l=1 \mathrm{~m}, E=207 \times 10^{9} \mathrm{~N} / \mathrm{m}^{2}, G=$ $77.6 \times 10^{9} \mathrm{~N} / \mathrm{m}^{2}, k=0.9, \rho=7700 \mathrm{~kg} / \mathrm{m}^{3}, m=0.2 \rho A l \mathrm{~kg}$, $k_{1}=10^{4} \mathrm{~N} / \mathrm{m}^{2}, \alpha=0.11, g=9.81 \mathrm{~m} / \mathrm{s}^{2}$, and $\beta=0.03$, in which $\alpha$ and $\beta$ are the velocity ratio and Rayleigh's slenderness coefficient, respectively. Note that $\beta=\pi r_{0} / l$ with $r_{0}$ taken as the beam's radius of gyration and $\alpha=v / v_{c r}$ with $v_{c r}$ as the critical velocity of a concentrated moving force defined as $v_{c r}=\omega_{1} l / \pi[9]$, with $\omega_{1}$ as the first natural angular frequency of transversal vibration of this beam given in general as $\left(\omega_{i}\right)=$ $(i \pi / l)^{2} \sqrt{E I_{d} / \rho A}(i=1,2, \ldots, n)[9,10]$. In addition, it can be seen that the critical velocity for the first mode $(i=1)$ is given as $v_{c r}=(\pi / l) \sqrt{E I_{d} / \rho A}[9,10]$.

Based on the above data, the computer code was run for this case, and the normalized instantaneous lateral displacement $\left(w_{p} / w_{s}\right)$ of the instantaneous positions of the moving mass $(x / l)$ is calculated and the dimensionless outcome results are depicted and compared with other existing results in Figure 2. The normalization parameter for the lateral displacement, that is, $w_{s}$, is actually a midpoint deflection of a simply supported beam under midspan concentrated load of $m g$; that is, $w_{s}=m g l^{3} / 48 E I_{d}[9,10]$. A close inspection of the curves in Figure 2 indicates perfect agreements between the two outcome results.

4.1.2. Nonlinear Analysis. A simply supported Timoshenko beam resting on a Pasternak-type viscoelastic and a shear foundation and traveled by a constant velocity moving force as shown in Figure 3 is considered. For this system, (5) and (6) are rewritten as follows:

$$
\begin{aligned}
& \rho A u_{, t t}-E A\left(u_{, x x}+w_{, x} w_{, x x}\right)=0, \\
& \rho A w_{, t t}+k G A\left(\Gamma_{, x}-w_{, x x}\right) \\
&-E A\left(u_{, x x} w_{, x}+u_{, x} w_{, x x}+\frac{3}{2} w_{, x x} w_{, x}^{2}\right) \\
&-k_{p} w_{, x x}+k_{1} w+k_{3} w^{3}+c w_{, t} \\
&=--P \delta(x-\zeta(t)) \chi(t),
\end{aligned}
$$




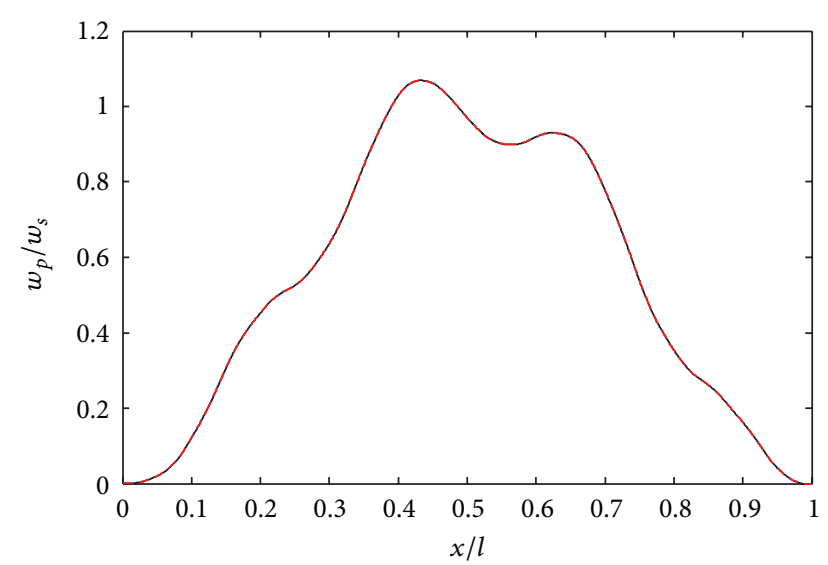

FIGURE 2: Instantaneous normalized lateral displacement $w_{p} / w_{s}$ under a moving mass of $m=0.2 \rho A l \mathrm{~kg}, k_{1}=10^{4} \mathrm{~N} / \mathrm{m}^{2}, \alpha=0.11$, and $\beta=0.03$; (-) linear analysis, present study, (.....) linear analysis, [9].

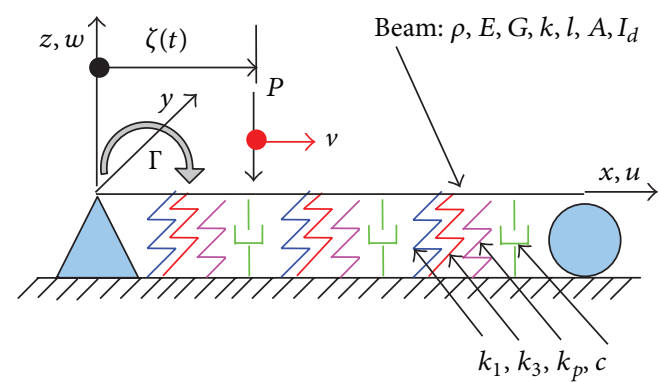

FIgURE 3: A simply supported Timoshenko beam resting on a nonlinear Pasternak viscoelastic foundation and subjected to a moving force $P$ (see [17]).

in which $k_{p}$ and $P$ are Pasternak or shear foundation modulus and the value of moving force, respectively, and (7) remains unchanged.

To check on the validity of our solutions based on (7) and (15), we consider the reported results on [17] in which analyses shear deformable beams on tensionless nonlinear Pasternak-type viscoelastic foundations and subjected to moving forces. The following data, that is, $l=10 \mathrm{~m}, I_{d}=$ $39.5 \times 10^{-6} \mathrm{~m}^{4}, A=86.13 \times 10^{-4} \mathrm{~m}^{2}, E=207 \times 10^{9} \mathrm{~N} / \mathrm{m}^{2}, G=$ $79.61 \times 10^{9} \mathrm{~N} / \mathrm{m}^{2}, k=0.85, \rho=7820 \mathrm{~kg} / \mathrm{m}^{3}, k_{1}=20 \times$ $10^{6} \mathrm{~N} / \mathrm{m}^{2}, k_{3}=0 \mathrm{~N} / \mathrm{m}^{4}, k_{p}=69 \times 10^{3} \mathrm{~N}, c=138 \times 10^{3} \mathrm{~N} \cdot \mathrm{s} / \mathrm{m}^{2}$, $P=144 \times 10^{3} \mathrm{~N}$, and $v=16.7 \mathrm{~m} / \mathrm{s}$, are adapted from [17]. Based on above mentioned equations, a computer program was developed and the outcome nonlinear results are depicted and compared with [17] in Figure 4. A close inspection of the curves reveals very close agreements between the two solutions.

4.2. Results and Discussions. After being satisfied with the validity of solution technique, the beam's instantaneous dynamic lateral deflection is calculated in the next step. In

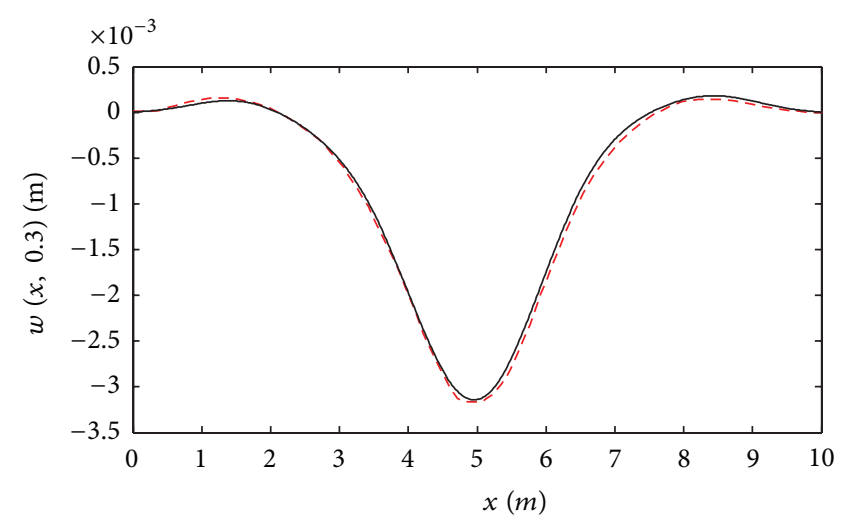

Figure 4: Lateral displacement $w(x, 0.3)$ at instant $t=0.3 \mathrm{~s}$ along length of the beam; (-) nonlinear analysis, present study, (- - - -) nonlinear analysis, [17].

obtaining these results the following data [9] are taken into consideration, unless otherwise specified:

$$
\begin{aligned}
& l=1 \mathrm{~m}, \quad E=207 \times 10^{9} \mathrm{~N} / \mathrm{m}^{2}, \quad G=77.6 \times 10^{9} \mathrm{~N} / \mathrm{m}^{2}, \\
& k=0.9, \quad \rho=7700 \mathrm{~kg} / \mathrm{m}^{3}, \quad I_{d}=1.045 \times 10^{-3} \mathrm{~m}^{4}, \\
& A=0.1146 \mathrm{~m}^{2}, \quad m=3 \rho A l \mathrm{~kg}, \quad k_{1}=10^{4} \mathrm{~N} / \mathrm{m}^{2}, \\
& k_{3}=10^{19} \mathrm{~N} / \mathrm{m}^{4}, \quad c=10^{3} \mathrm{~N} \cdot \mathrm{s} / \mathrm{m}^{2}, \quad \beta=0.3 .
\end{aligned}
$$

It should be mentioned that all deflection variations versus moving mass instantaneous positions are given in a nondimensional form, that is, $w_{p} / w_{s}$. Moreover, it has to be pointed out that based on the conducted convergence study related to the linear and nonlinear analyses, ten modes of vibration are taken into account for the steady state answers for $u(x, t), w(x, t)$, and $\Gamma(x, t)$; that is, $n=10$.

The effect of variation of nonlinear part of foundation stiffness, $k_{3}$, and geometrical nonlinearity due to the beam's end boundary conditions on the dynamic responses of a Timoshenko beam traversed by a moving mass are depicted in Figure 5. In this analysis $m=3 \rho A l \mathrm{~kg}, k_{1}=10^{4} \mathrm{~N} / \mathrm{m}^{2}, c=$ $10^{3} \mathrm{~N} \cdot \mathrm{s} / \mathrm{m}^{2}$, and $\beta=0.3$ and different speeds for the traveling mass $(\alpha=0.11,0.25$, and 0.5$)$ are considered. It should be noted that, under adopted data, the results for M-2 and M-3 models are similar and, hence, the variations are overlaid. This means that as the beam gets thicker it resists more against the stretching of midplane surface. Therefore, stretching does not play an important role in the beam's dynamical response especially. In addition, in this figure the differences between dynamical behavior of the beam between M-2 or M-3 models and M-1 model are compared with each other. Out of this figure and as it is expected, it can be concluded that by increasing the value of $k_{3}$ the difference between M-2 or M-3 models and M-1 model becomes more pronounced. In other words, by including geometrical and foundation nonlinearities into the M-1 model as the reference beam, as the size of nonlinearity increases the difference of the values of $w_{p} / w_{s}$ 


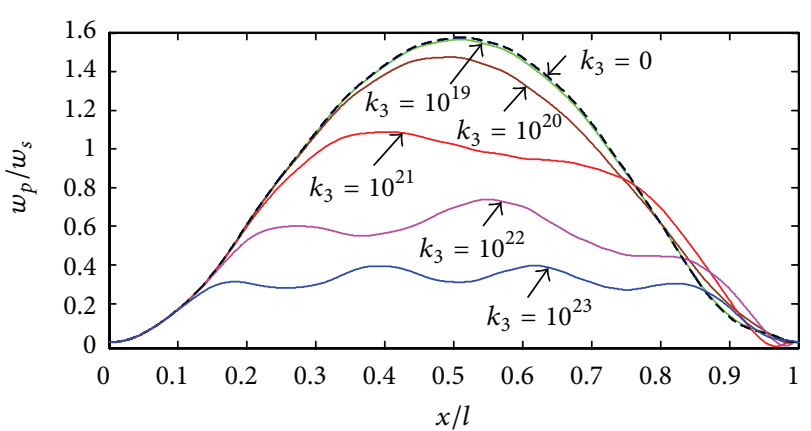

(a)

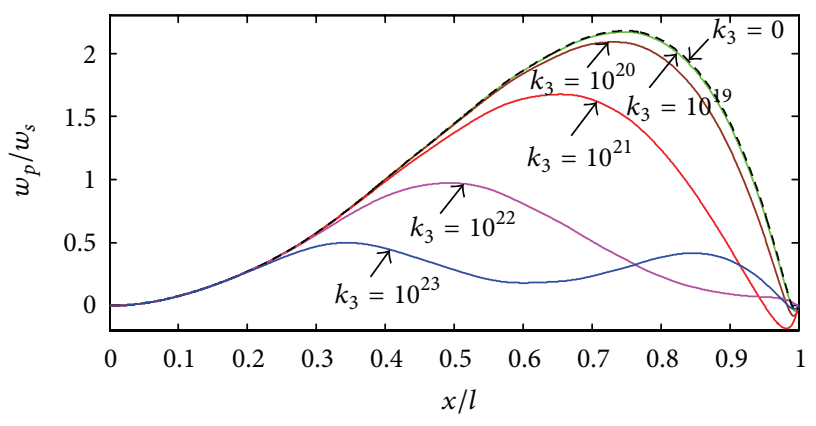

(b)

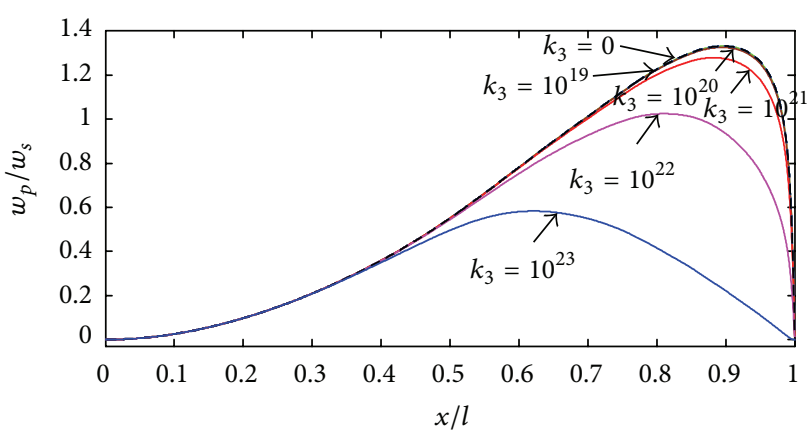

(c)

FIGURE 5: Variation of dimensionless instantaneous dynamic response, $w_{p} / w_{s}$, versus normalized instantaneous position of moving mass, $x / l$, of the Timoshenko beam with different $k_{3}$ values traveled at different speeds with $m=3 \rho A l \mathrm{~kg}, k_{1}=10^{4} \mathrm{~N} / \mathrm{m}^{2}$, $c=10^{3} \mathrm{~N} \cdot \mathrm{s} / \mathrm{m}^{2}$, and $\beta=0.3$. (a) $\alpha=0.11$, (b) $\alpha=0.25$, (c) $\alpha=0.5$; (- - - -) M-1 model, (-) M-2 model, or M-3 model.

in the nonlinear models (M-2 or M-3) compared to the M-1 model increases. This difference becomes more pronounced for $k_{3}=10^{23} \mathrm{~N} / \mathrm{m}^{4}$. Furthermore, when the mass velocity increases, the maximum value of the beam's dynamic response increases up to $\alpha=0.25$ and decreases afterward. In addition, it can be seen that for the $k_{3}$ values greater than $10^{21} \mathrm{~N} / \mathrm{m}^{4}$, by increasing the value of $\alpha$, the fluctuation of dynamical response of the beam reduces accordingly and finally, for $k_{3}=10^{23} \mathrm{~N} / \mathrm{m}^{4}$ and $\alpha=0.11$, this fluctuation reaches its highest value.

Figure 6 shows the variation of normalized lateral dynamic displacement, $w_{p} / w_{s}$, versus normalized instantaneous position of moving mass, $x / l$, for three different Timoshenko beams of $\mathrm{M}-1, \mathrm{M}-2$, and $\mathrm{M}-3$ traversed by a moving

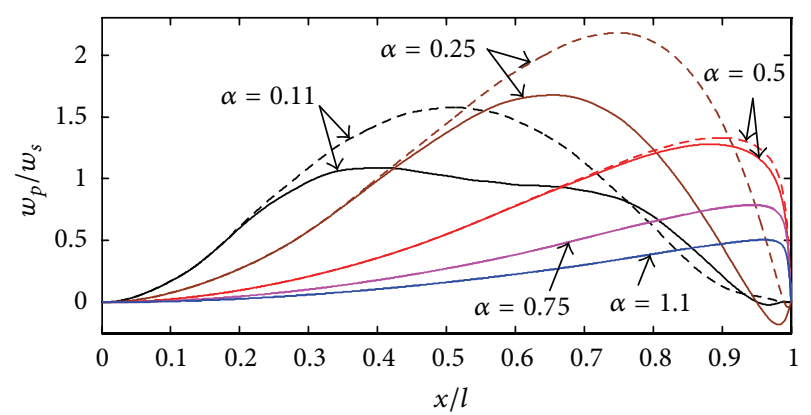

FIGURE 6: Variation of dimensionless instantaneous dynamic lateral deflection, $w_{p} / w_{s}$, versus normalized instantaneous position of moving mass, $x / l$, of the Timoshenko beam traveled at different speeds with $m=3 \rho A l \mathrm{~kg}, k_{1}=10^{4} \mathrm{~N} / \mathrm{m}^{2}, c=10^{3} \mathrm{~N} \cdot \mathrm{s} / \mathrm{m}^{2}$, and $\beta=0.3$; ( - . -) M-1 model, (-) M-2, or M-3 model with $k_{3}=10^{21} \mathrm{~N} / \mathrm{m}^{4}$.

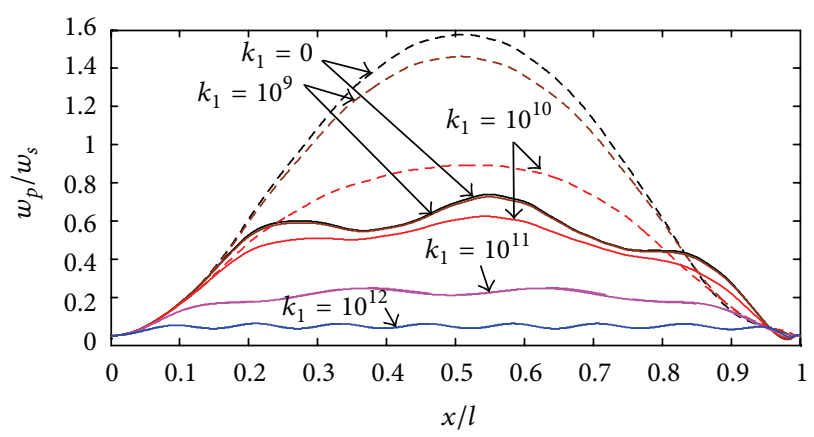

(a)

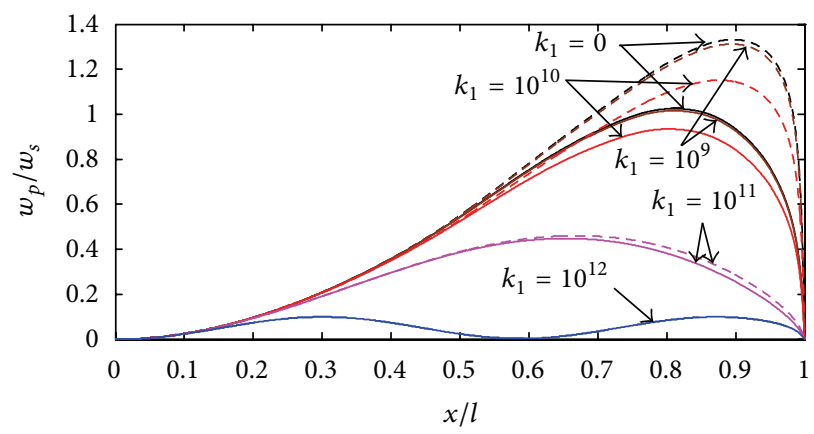

(b)

FIGURE 7: Variation of dimensionless instantaneous dynamic lateral deflection, $w_{p} / w_{s}$, versus normalized instantaneous position of moving mass, $x / l$, of the Timoshenko beam with different $k_{1}$ values traveled at two different speeds with $m=3 \rho A l \mathrm{~kg}, c=10^{3} \mathrm{~N} \cdot \mathrm{s} / \mathrm{m}^{2}$, and $\beta=0.3$. (a) $\alpha=0.11$, (b) $\alpha=0.5$; (- - - ) M-1 model and (-) M-2 or M-3 model with $k_{3}=10^{22} \mathrm{~N} / \mathrm{m}^{4}$.

mass. The following data are considered: $m=3 \rho A l \mathrm{~kg}$, $k_{1}=10^{4} \mathrm{~N} / \mathrm{m}^{2}, k_{3}=10^{21} \mathrm{~N} / \mathrm{m}^{4}, c=10^{3} \mathrm{~N} \cdot \mathrm{s} / \mathrm{m}^{2}$, and $\beta=0.3$. Moreover, different speeds for the traveling mass are considered $(\alpha=0.11,0.25,0.5,0.75$, and 1.1). It can be seen from this figure that the $w_{p} / w_{s}$ results for the M-2 or M-3 models are always lower than the one obtained from the linear beam model, that is, M-1 model. However, the $w_{p} / w_{s}$ results for M2 and M-3 are almost the same and independent of the value 


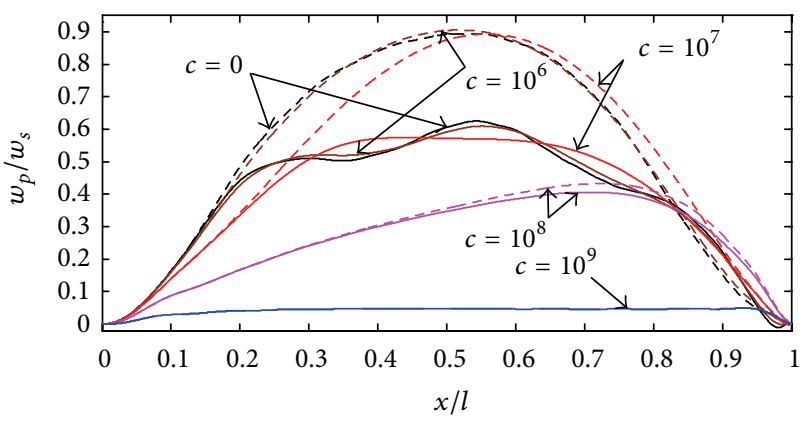

(a)

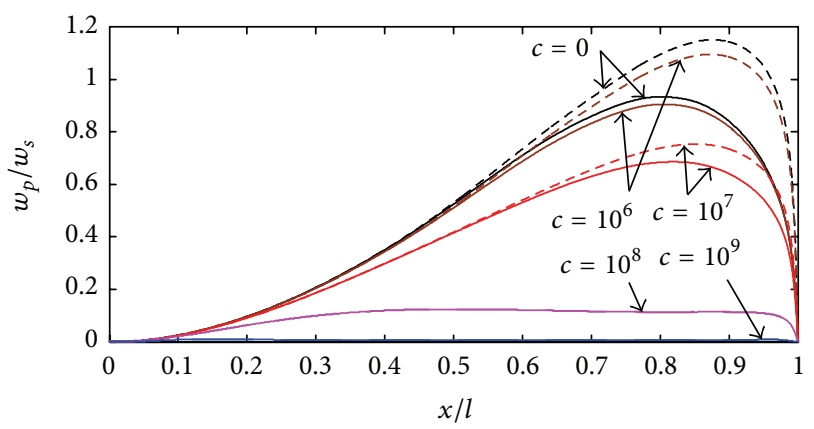

(b)

FIGURE 8: Variation of dimensionless instantaneous dynamic lateral deflection, $w_{p} / w_{s}$, versus normalized instantaneous position of moving mass, $x / l$, of the Timoshenko beam with different $c$ values traveled at two different speeds with $m=3 \rho A l \mathrm{~kg}, k_{1}=10^{10} \mathrm{~N} / \mathrm{m}^{2}$, and $\beta=0.3$. (a) $\alpha=0.11$, (b) $\alpha=0.5$; ( - - - ) M-1 model and (-) M-2 or M-3 model with $k_{3}=10^{22} \mathrm{~N} / \mathrm{m}^{4}$.

of velocity ratio. The maximum difference between $M-1$ and M-2 or M-3 models always happens at $\alpha=0.25$. Furthermore, it can be seen that in this case the maximum lateral dynamic deflection $w_{p} / w_{s}$ increases up to $\alpha=0.25$ and a reversing trend prevails afterward no matter what types of beam models are used.

Using linear (M-1 model) and nonlinear (M-2 and M-3 models) Timoshenko beam models, Figure 7 shows the variation of normalized lateral dynamic displacement, $w_{p} / w_{s}$, versus normalized instantaneous position of moving mass, $x / l$. As it was explained before these beams are resting on a viscoelastic foundation. For further analysis different values of $k_{1}$ are considered for these beam models. The following data are chosen: $k_{3}=10^{22} \mathrm{~N} / \mathrm{m}^{4}, c=10^{3} \mathrm{~N} \cdot \mathrm{s} / \mathrm{m}^{2}$, and $\beta=0.3$, with mass moving at two different speeds $(\alpha=0.11$ and 0.5$)$. It can be seen from this figure that the results of $w_{p} / w_{s}$ obtained from the M-2 and M-3 models with $k_{3}=10^{22} \mathrm{~N} / \mathrm{m}^{4}$ are the same and always lower than the one obtained from the M-1 model, where the maximum difference between M-1 and M-2 or M-3 models always happens at $k_{1}=0$ and is independent of the value of mass velocity. Also, when the value of $k_{1}$ increases, the difference between M-1 and M-2 or M-3 models decreases accordingly. For example, when $k_{1}=$ $10^{11} \mathrm{~N} / \mathrm{m}^{2}$, this difference becomes negligible. Furthermore, by comparing Figure 7(a) with Figure 7(b), it can be observed that the beam's nonlinear dynamic response for M-2 and M-3

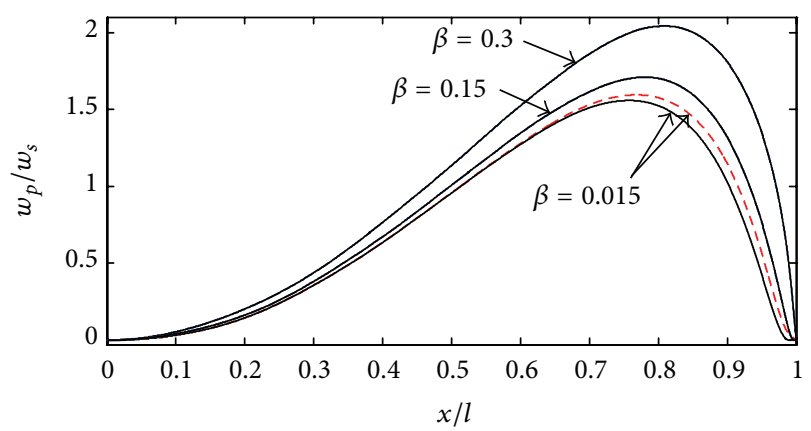

(a)

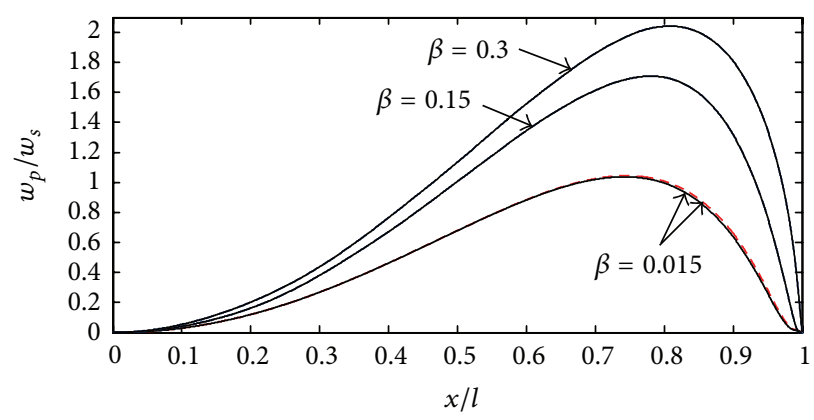

(b)

FIGURE 9: Variation of dimensionless instantaneous dynamic lateral deflection, $w_{p} / w_{s}$, versus normalized instantaneous position of moving mass, $x / l$, of the Timoshenko beam with $m=5 \rho A l \mathrm{~kg}$ and $\alpha=0.25$ and different $\beta$ values. (a) $k_{1}=k_{3}=c=0$, (b) $k_{1}=10^{4} \mathrm{~N} / \mathrm{m}^{2}, k_{3}=10^{4} \mathrm{~N} / \mathrm{m}^{4}$, and $c=10^{3} \mathrm{~N} \cdot \mathrm{s} / \mathrm{m}^{2} ;(-\ldots)$ - - $\mathrm{M}-1$ model and (-) M-2 or M-3 model.

models at $\alpha=0.5$ is greater than the one related to $\alpha=0.11$. Nevertheless, a reversing trend is seen for the beam's M-1 model dynamic response.

Figure 8 shows the variation of normalized lateral dynamic displacement, $w_{p} / w_{s}$, versus normalized instantaneous position $x / l$ for three different Timoshenko beams of M-1, M-2, and M-3 traversed by a moving mass. In this case different values of $c$ are considered. The following data are chosen: $m=3 \rho A l \mathrm{~kg}, k_{1}=10^{10} \mathrm{~N} / \mathrm{m}^{2}, k_{3}=10^{22} \mathrm{~N} / \mathrm{m}^{4}$, and $\beta=0.3$, with mass moving at two different speeds $(\alpha=$ 0.11 and 0.5 ). It can be seen from this figure that the beams dynamic responses of $w_{p} / w_{s}$ obtained from the M-2 or M3 models are always lower than the one obtained from the M-1 model, where the maximum difference between $M$ 1 and M-2 or M-3 models always happens at $c=0$ no matter what values of mass velocity would be. Also, when the value of $c$ increases the beam's dynamical response difference between M-1 and M-2 or M-3 models decreases accordingly, where for $c=10^{9} \mathrm{~N} \cdot \mathrm{s} / \mathrm{m}^{2}$ this difference becomes negligible. Furthermore, by comparing Figure 8(a) with Figure 8(b) it can be concluded that for $c<10^{8} \mathrm{~N} \cdot \mathrm{s} / \mathrm{m}^{2}$ the obtained beam's dynamic response at $\alpha=0.5$ is almost greater than the one obtained at $\alpha=0.11$ no matter what type of beam models is used.

Figure 9 shows the variation of normalized lateral dynamic displacement, $w_{p} / w_{s}$, versus normalized instantaneous position $x / l$ for three different Timoshenko beams of 
M-1, M-2, and M-3. The following data are assumed: $m=$ $5 \rho A l \mathrm{~kg}$ and mass traveling speed is $\alpha=0.25$. The results are presented for three different slenderness ratios $(\beta)$. Furthermore, it should be noted that in Figure 9(a) $k_{1}=k_{3}=c=$ 0 and in Figure 9(b) $k_{1}=10^{4} \mathrm{~N} / \mathrm{m}^{2}, k_{3}=10^{4} \mathrm{~N} / \mathrm{m}^{4}$, and $c=$ $10^{3} \mathrm{~N} \cdot \mathrm{s} / \mathrm{m}^{2}$ are considered. It can be seen from this figure that the maximum value of dynamical response, $w_{p} / w_{s}$, increases as $\beta$ increases. Moreover, the position of maximum value of $w_{p} / w_{s}$ moves to the right end of the beam as $\beta$ increases. It can be concluded from this figure that inclusion of nonlinear foundation parameters will decrease the difference of linear and nonlinear analyses. It should be further mentioned that the results for linear and nonlinear analyses are the same for $\beta=0.15$ and 0.3 .

\section{Conclusions}

The longitudinal and lateral deflections as well as the rotation of warped cross section of three different Timoshenko beams of M-1, M-2, and M-3 models resting on viscoelastic foundation and subjected to a moving mass with constant velocity are all considered by including the nonlinear nature in the beam's geometry and the stiffness of viscoelastic foundation. The outcome results are as the following.

(1) It is concluded that by including geometrical and foundation nonlinearities into the reference M-1 model, as the size of nonlinearity increases the difference of the beam's dynamic response in the nonlinear models (M-2 or M-3) and M-1 model increases. This difference becomes more pronounced for the comparatively large enough values of nonlinear stiffness part of viscoelastic foundation.

(2) It is observed that the beam's dynamic response results for the fully nonlinear beam model, that is, M-3 model, are always lower than the one obtained from the linear beam model, that is, M-1 model.

(3) It is concluded that when the value of linear stiffnesspart of foundation increases, the difference between M-1 and M-2 or M-3 models decreases accordingly, where forthe large values of linear stiffness part of foundation this difference becomes negligible. Moreover, when the value of foundation damping increases the beam's dynamical response difference between $\mathrm{M}$ 1 and M-2 or M-3 models decreases accordingly.

(4) Because both stretching effect and nonlinear stiffness part of viscoelastic foundation in conjunction with moving mass condition and the other values of the foundation's parameters are significant factors in the nonlinear dynamic behavior of the beam, with changing the beam's slenderness ratio $\beta$ it appears that for $\beta \leq 0.015$ nonlinear behavior predominantly can be seen. However, for $\beta>0.015$ no specific values for the critical value of the traveling mass $m$ and foundation nonlinear stiffness $k_{3}$ were obtained which could be an idea for further investigation.

\section{Appendix}

The definition of different matrices used in calculation of the nonlinear coupled ODEs of modal relations (11) is as

$$
\begin{gathered}
(\mathbf{M})_{i j}=\int_{0}^{l} \phi_{i}(x) \phi_{j}(x) d x, \quad(\mathbf{H})_{i j}=\int_{0}^{l} \phi_{i}(x) \phi_{j}^{\prime \prime}(x) d x, \\
(\mathbf{F})_{i j}=\int_{0}^{l} \phi_{i}(x) \psi_{j}^{\prime}(x) d x, \quad(\mathbf{S})_{i j}=\int_{0}^{l} \psi_{i}(x) \psi_{j}(x) d x, \\
(\mathbf{K})_{i j}=\int_{0}^{l} \psi_{i}(x) \psi_{j}^{\prime \prime}(x) d x, \quad(\mathbf{E})_{i j}=\int_{0}^{l} \psi_{i}(x) \phi_{j}^{\prime}(x) d x, \\
(\mathbf{J})_{i j}=\int_{0}^{l} \theta_{i}(x) \theta_{j}(x) d x, \quad(\mathbf{N})_{i j}=\int_{0}^{l} \theta_{i}(x) \theta_{j}^{\prime \prime}(x) d x, \\
(\mathbf{G})_{i j k}=\int_{0}^{l} \phi_{i}(x) \theta_{j}^{\prime \prime}(x) \phi_{k}^{\prime}(x) d x, \\
(\mathbf{I})_{i j k}=\int_{0}^{l} \phi_{i}(x) \phi_{j}^{\prime \prime}(x) \phi_{k}^{\prime 2}(x) d x, \\
(\mathbf{L})_{i j k}=\int_{0}^{l} \theta_{i}(x) \phi_{j}^{\prime \prime}(x) \phi_{k}^{\prime}(x) d x, \\
\left(\mathbf{R}_{1}\right)_{i j k}=\int_{0}^{l} \phi_{i}(x) \phi_{j}^{\prime \prime}(x) \theta_{k}^{\prime}(x) d x, \\
\left(\mathbf{R}_{2}\right)_{i j k l}=\int_{0}^{l} \phi_{i}(x) \phi_{j}(x) \phi_{k}(x) \phi_{l}(x) d x, \\
(\mathbf{A})_{i j}=\phi_{i}(x=\zeta(t)) \phi_{j}^{\prime}(x=\zeta(t)), \\
(\mathbf{B})_{i j}=\phi_{i}(x=\zeta(t)) \phi_{j}(x=\zeta(t)), \\
(\mathbf{C})_{i j}=\phi_{i}(x=\zeta(t)) \phi_{j}^{\prime \prime}(x=\zeta(t)), \\
(\mathbf{O})_{i j}=\theta_{i}(x=\zeta(t)) \theta_{j}(x=\zeta(t)),
\end{gathered}
$$

where $i, j, k, l=1,2,3, \ldots, n$, in which prime mark over any parameter indicates the derivative with respect to the position $(x)$. Furthermore, the vector of $\mathbf{a}$ in the form of $n \times 1$ column is given by

$$
\phi_{i}(x=\zeta(t))=(\mathbf{a})_{i} .
$$

\section{Conflict of Interests}

The authors declare that there is no conflict of interests regarding the publication of this paper.

\section{Acknowledgment}

This paper is dedicated to Ahmad Mamandi's dearest Professor Dr. Mohammad Hossein Kargarnovin, passed away on Monday, November 4, 2013. He was a fine, kind, decent, sincere, good tempered, and well-liked professor who will be sadly missed by all who knew him. May his soul rest in peace. 


\section{References}

[1] D. Thambiratnam and Y. Zhuge, "Dynamic analysis of beams on an elastic foundation subjected to moving loads," Journal of Sound and Vibration, vol. 198, no. 2, pp. 149-169, 1996.

[2] Y.-H. Chen, Y.-H. Huang, and C.-T. Shih, "Response of an infinite tomoshenko beam on a viscoelastic foundation to a harmonic moving load," Journal of Sound and Vibration, vol. 241, no. 5, pp. 809-824, 2001.

[3] S.-M. Kim and Y.-H. Cho, "Vibration and dynamic buckling of shear beam-columns on elastic foundation under moving harmonic loads," International Journal of Solids and Structures, vol. 43, no. 3-4, pp. 393-412, 2006.

[4] C. R. Steele, "The finite beam with a moving load," Journal of Applied Mechanics, vol. 34, pp. 111-118, 1967.

[5] A. L. Florence, "Traveling force on a Timoshenko beam," Journal of Applied Mechanics, vol. 32, pp. 351-358, 1965.

[6] H. D. Nelson and R. A. Conover, "Dynamic stability of a beam carrying moving masses," Journal of Applied Mechanics, vol. 38, no. 4, pp. 1003-1006, 1971.

[7] T.-P. Chang and Y.-N. Liu, "Dynamic finite element analysis of a nonlinear beam subjected to a moving load," International Journal of Solids and Structures, vol. 33, no. 12, pp. 1673-1688, 1996.

[8] Y. C. Hou, C. H. Tseng, and S. F. Ling, "A new high-order non-uniform Timoshenko beam finite element on variable twoparameter foundations for vibration analysis," Journal of Sound and Vibration, vol. 191, no. 1, pp. 91-106, 1996.

[9] H. P. Lee, "Dynamic response of a Timoshenko beam on a winkler foundation subjected to a moving mass," Applied Acoustics, vol. 55, no. 3, pp. 203-215, 1998.

[10] P. Lou, G.-L. Dai, and Q.-Y. Zeng, "Finite-element analysis for a Timoshenko beam subjected to a moving mass," Proceedings of the Institution of Mechanical Engineers, Part C: Journal of Mechanical Engineering Science, vol. 220, no. 5, pp. 669-678, 2006.

[11] Y.-H. Chen and J.-T. Sheu, "Axially-loaded damped Timoshenko beam on viscoelastic foundation," International Journal for Numerical Methods in Engineering, vol. 36, no. 6, pp. 10131027, 1993.

[12] Z. Hryniewicz and P. Koziol, "Wavelet-based solution for vibrations of a beam on a nonlinear viscoelastic foundation due to moving load," Journal of Theoretical and Applied Mechanics, vol. 51, no. 1, pp. 215-224, 2013.

[13] P. Koziol and Z. Hryniewicz, "Dynamic response of a beam resting on a nonlinear foundation to a moving load: coiflet-based solution," Shock and Vibration, vol. 19, pp. 995-1007, 2012.

[14] Z. Hryniewicz, "Dynamics of Rayleigh beam on nonlinear foundation due to moving load using Adomian decomposition and coiflet expansion," Soil Dynamics and Earthquake Engineering, vol. 31, no. 8, pp. 1123-1131, 2011.

[15] Z. Dimitrovová, "A general procedure for the dynamic analysis of finite and infinite beams on piece-wise homogeneous foundation under moving loads," Journal of Sound and Vibration, vol. 329, no. 13, pp. 2635-2653, 2010.

[16] V.-H. Nguyen and D. Duhamel, "Finite element procedures for nonlinear structures in moving coordinates. Part II: infinite beam under moving harmonic loads," Computers and Structures, vol. 86, no. 21-22, pp. 2056-2063, 2008.

[17] E. J. Sapountzakis and A. E. Kampitsis, "Nonlinear response of shear deformable beams on tensionless nonlinear viscoelastic foundation under moving loads," Journal of Sound and Vibration, vol. 330, no. 22, pp. 5410-5426, 2011.
[18] T. Mazilu, M. Dumitriu, and C. Tudorache, "Instability of an oscillator moving along a Timoshenko beam on viscoelastic foundation," Nonlinear Dynamics, vol. 67, no. 2, pp. 1273-1293, 2012.

[19] L. Meirovitch, Principles and Techniques of Vibrations, PrenticeHall, Prentice, NJ, USA, 1997.

[20] A. Mamandi, M. H. Kargarnovin, and S. Farsi, "An investigation on effects of traveling mass with variable velocity on nonlinear dynamic response of an inclined Timoshenko beam with different boundary conditions," International Journal of Mechanical Sciences, vol. 52, no. 12, pp. 1694-1708, 2010.

[21] A. Mamandi and M. H. Kargarnovin, "Nonlinear dynamic analysis of an inclined Timoshenko beam subjected to a moving mass/force with beam's weight included," Shock and Vibration, vol. 18, no. 6, pp. 875-891, 2011. 

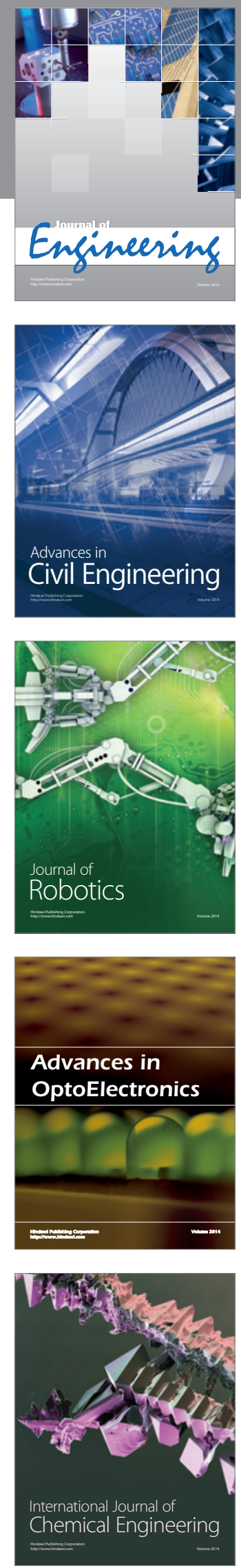

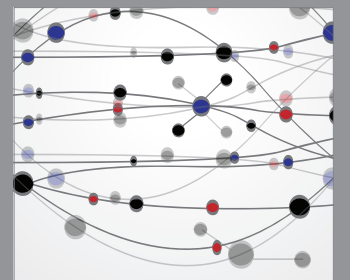

The Scientific World Journal
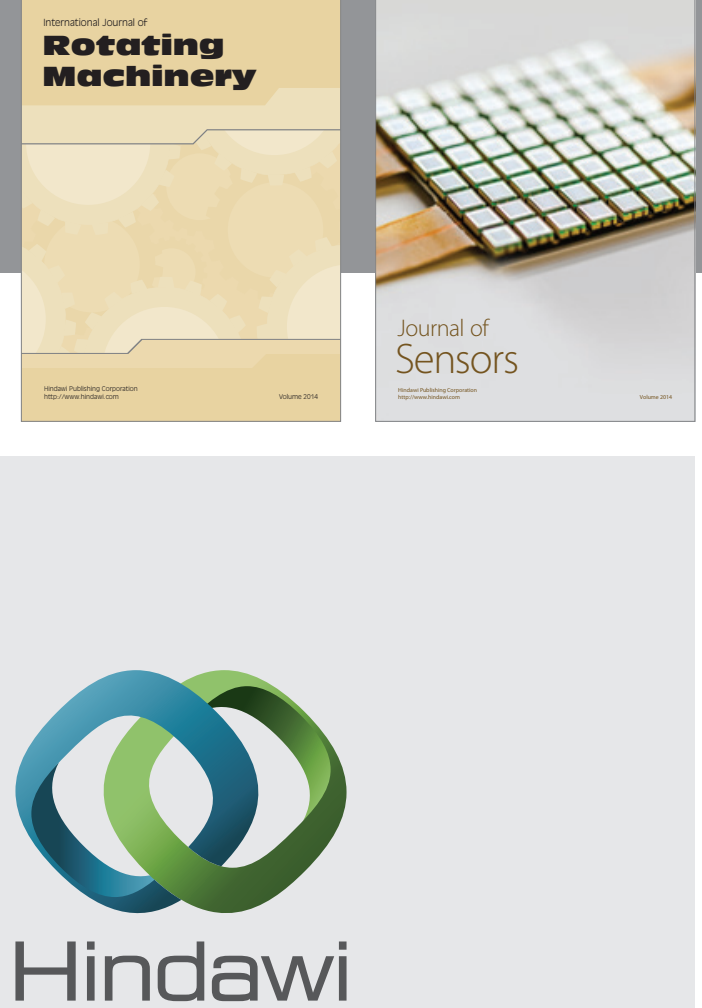

Submit your manuscripts at http://www.hindawi.com
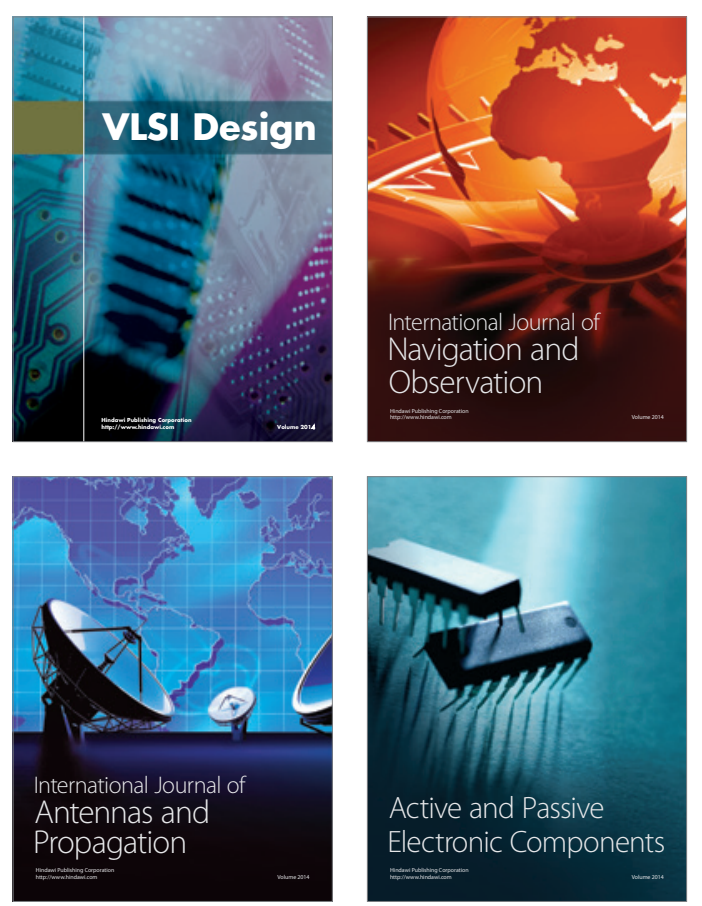
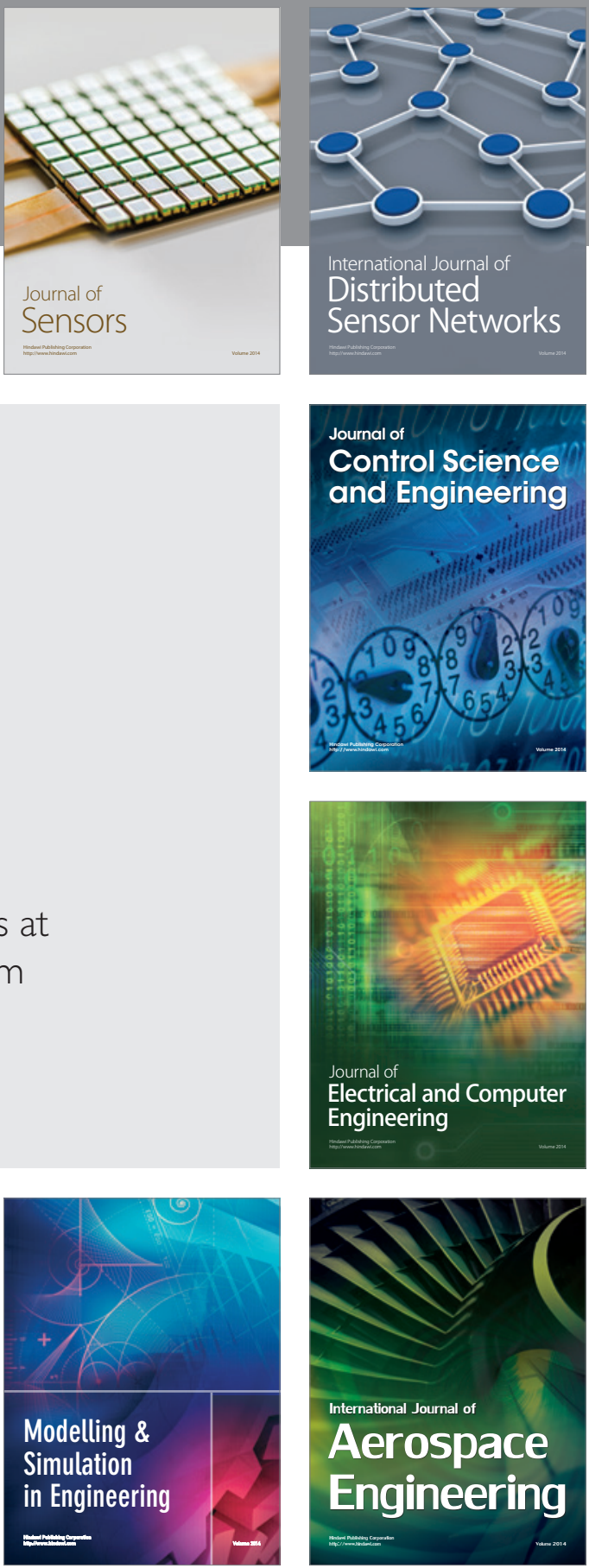

Journal of

Control Science

and Engineering
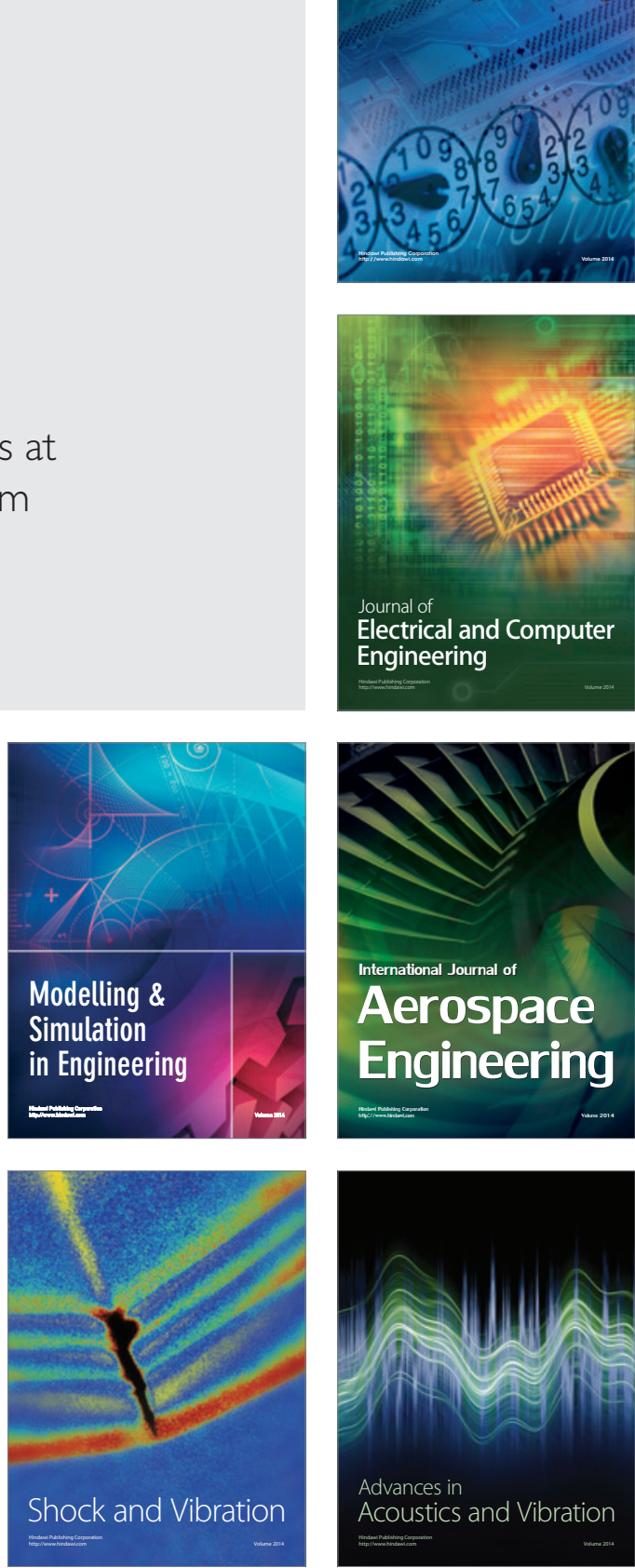